\title{
Combined Endoscopic and External Surgical Approach for Lateralization of Vocal Cord in Bilateral Abductor Palsy using Suture Technique: Our Experience
}

\author{
${ }^{1}$ Ramesh G Babu, ${ }^{2}$ Krishna V Chaitanya, ${ }^{3}$ Lokesh P Kumar
}

\begin{abstract}
Introduction: Bilateral vocal cord paralysis is a rare but dangerous condition which results in shortness of breath and poor quality of voice. Only patients with severe bilateral vocal cord immobility require surgical intervention. In the present study, we describe the procedure of combined endoscopic and external surgical approach for lateralization of vocal cord in bilateral abductor palsy.
\end{abstract}

Objective: The objective of this study is to analyze the surgical outcome of combined endoscopic and external surgical approach for lateralization of vocal cord using suture technique in bilateral abductor palsy.

Materials and methods: An observational study was conducted on 17 patients presenting in ENT Department during May 2014 to July 2016 with laryngeal lesions of bilateral abductor palsy with posterior glottic chink of $3 \mathrm{~mm}$ or less, stridor at rest, and significant limitation of physical activity due to airway narrowing.

Observations: In the present study, we have performed suture lateralization of vocal cords in 17 patients. Of these, 11 (64.70\%) were female and $5(29.41 \%)$ were male with slight female preponderance. Mean age of performing procedure was 39 years with the patients in age group of 18 to 60 years. When clinical etiology of the patients leading to bilateral vocal cord palsy was observed, patients presenting with postsurgical causes appeared to develop bilateral vocal cord palsy in $47.05 \%$ of patients. During the study, it was observed that improvement in respiration was adequate in $16(94.11 \%)$ patients, and voice quality was socially acceptable in $16(94.11 \%)$ patients.

Discussion and conclusion: Surgery of laterofixation can provide immediate and long-lasting adequate airway and can be considered as minimally invasive with minimal alterations to laryngeal mucosa and subsequent preservation of laryngeal functions. This procedure appears efficient in management of bilateral vocal cord palsy.

Keywords: Bilateral, Laterofixation, Tracheostomy, Vocal cord paralysis.

\footnotetext{
${ }^{1,3}$ Assistant Professor, ${ }^{2}$ Associate Professor

${ }^{1-3}$ Department of ENT, Head and Neck Surgery, Narayana Medical College \& Hospital, Nellore, Andhra Pradesh, India

Corresponding Author: Krishna V Chaitanya, Associate Professor, Department of ENT, Head and Neck Surgery Narayana Medical College \& Hospital, Nellore, Andhra Pradesh India, Phone: +919963337475, e-mail: drvkc17@gmail.com
}

How to cite this article: Babu RG, Chaitanya KV, Kumar LP. Combined Endoscopic and External Surgical Approach for Lateralization of Vocal Cord in Bilateral Abductor Palsy using Suture Technique: Our Experience. Int J Phonosurg Laryngol 2017;7(2):44-47.

Source of support: Nil

Conflict of interest: None

\section{INTRODUCTION}

Larynx constitutes fibrocartilaginous framework with muscles for voice production, prevention of aspiration during deglutition, and it acts as a leading pathway to lower respiratory tract. ${ }^{1}$ Laryngeal paralysis is associated with inability to cope up with the normal laryngeal functions. Bilateral vocal cord paralysis is a rare but dangerous condition which always results in shortness of breath associated with or without change of voice. ${ }^{2}$ Causes of vocal fold fixation differ in adults and in children. In adults, these include mechanical causes, inflammatory processes affecting the cricoarytenoid or larynx, malignancy, postsurgical effects, neurological causes, radiation injury, metabolic causes, and toxins. ${ }^{3}$

Patients with medical conditions rarely require surgical intervention because treatment of the underlying condition often improves airway compromise. Only the patients with severe bilateral vocal cord immobility require surgical intervention. ${ }^{4}$ The treatment of bilateral immobile vocal cords is a balance between phonation, airway, and swallowing. A wide range of techniques are nowadays used to enable the patient both to speak and to breathe without the need of permanent tracheostomy. The literature is less clear concerning the indications for the surgical intervention in these patients. For laterofixation of vocal cords and improvement of voice following bilateral vocal cord paralysis, a naïve approach of lateral fixation of vocal cords which combines an endolaryngeal together with an external approach can be performed. ${ }^{5}$

In the present study, we describe the procedure of combined endoscopic and external surgical approach for lateralization of vocal cord in bilateral abductor palsy. This method being technically simple and less traumatic than previous methods is expected to provide promising results with minimum complications. 


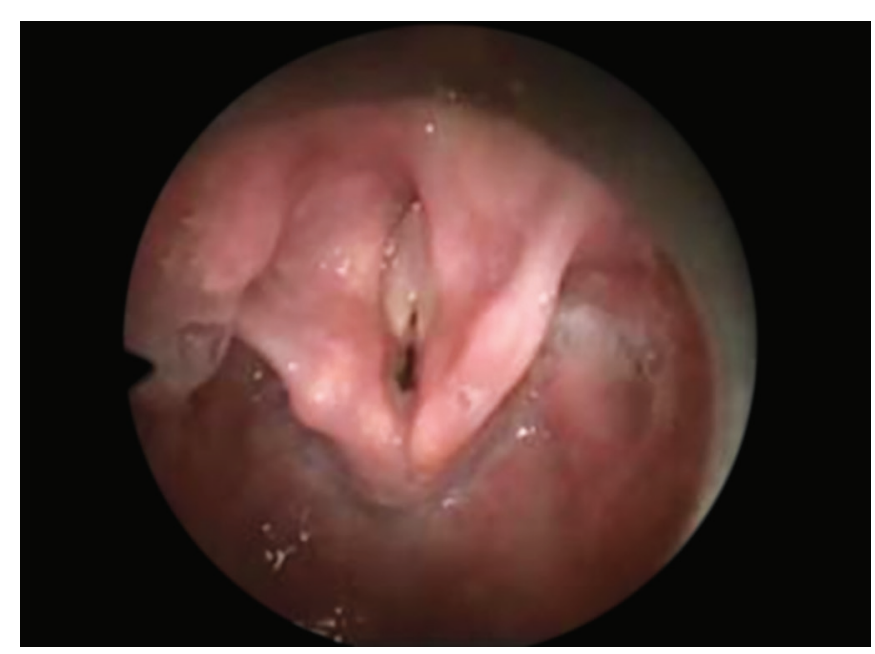

Fig. 1: Preoperative vocal cord status

\section{OBJECTIVES}

To study the surgical outcome of combined endoscopic and external surgical approach for lateralization of vocal cord using suture technique in bilateral abductor palsy.

\section{MATERIALS AND METHODS}

This prospective observational study was conducted on 17 patients presenting in ENT Department during May 2014 to July 2016 with laryngeal lesions of bilateral abductor palsy with posterior glottic chink of $3 \mathrm{~mm}$ or less, stridor at rest, and significant limitation of physical activity due to airway narrowing. Patients suffering with systemic granulomatous diseases, malignancies as confirmed by radiological investigations, were excluded from the study.

Detailed ear, nose, and throat examination was performed in all these patients. Radiological investigations including X-ray chest-posteroanterior view, X-ray soft tissue neck-anteroposterior and lateral views were performed in all the patients. All these patients were further evaluated with direct laryngoscopy using $70^{\circ}$ rigid Hopkins rod telescope, and the findings were confirmed. To confirm the findings, computed tomography (CT) scan of the neck and chest, ultrasonography of neck, magnetic resonance imaging of brain, CT scan of lateral skull base were performed as and where required.

All these patients were operated with combined endoscopic and external surgical approach using suture technique after obtaining an informed consent about the procedure. All the patients were operated under general anesthesia. The preferred modality of anesthesia management included endotracheal tube management. Tracheostomy was preferred as and where required for the completion of the procedure. All the surgeries were performed by a team of surgeons who had adequate experience in management of laryngeal diseases.

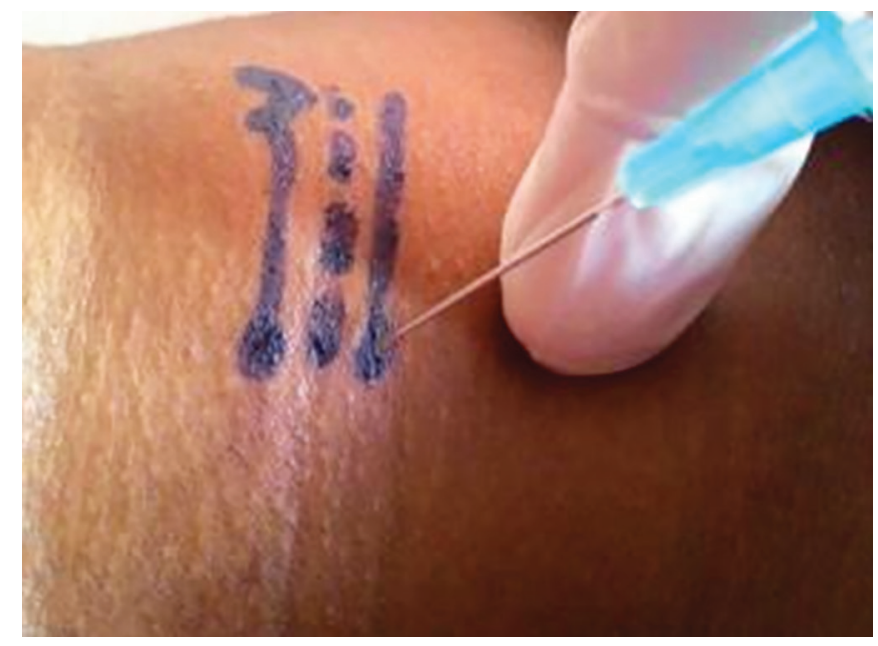

Fig. 2: External marking for wide-bore needle insertion

The procedure involves a combined endoscopic and external surgical approach which is simultaneously performed by two surgeons. After successful fixation of suspension laryngoscope, surgeon guides $0^{\circ}$ rigid Hopkins rod telescope into the glottic space exposing the larynx as shown in Figure 1.

Simultaneously, two needles are inserted through the thyroid cartilage using a wide-bore 18-gauge needle. First needle is inserted over thyroid cartilage 13 to $15 \mathrm{~mm}$ posterior to midline and 5 to $7 \mathrm{~mm}$ superior to inferior margins of thyroid cartilage. The second needle is inserted 2 to $3 \mathrm{~mm}$ below the first needle. The external markings for the insertion of wide-bore needle are shown in Figure 2.

The positions of needles were confirmed by endoscopy taking care to see one needle is above the vocal cords and one below the vocal cords; 3-0 Prolene was introduced into the lower needle from outside. This is pictorially observed in Figure 3. It was grasped by a microlaryngeal needle holder. It was then redirected to pass through the upper needle from within the airway to

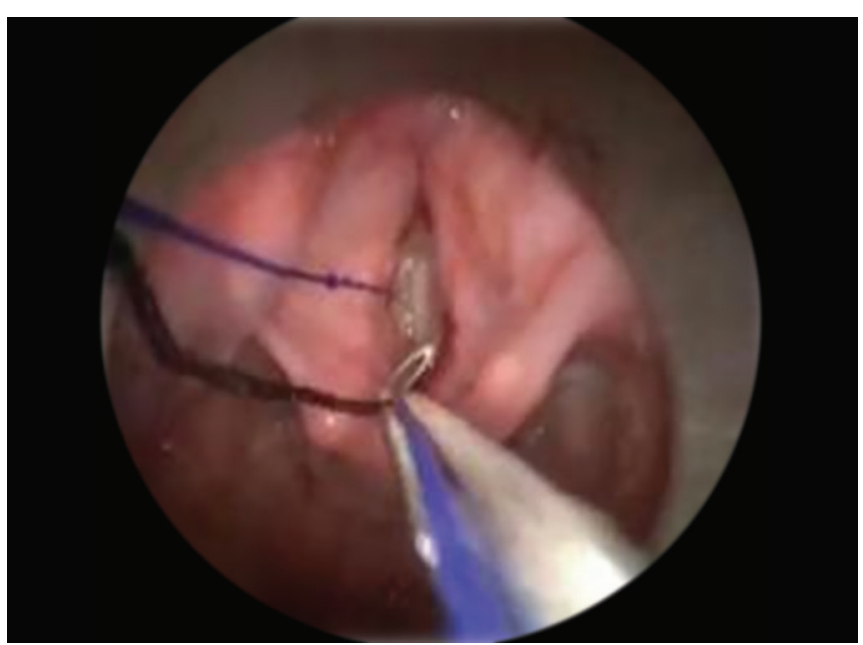

Fig. 3: Needle in place with 3-0 Prolene 


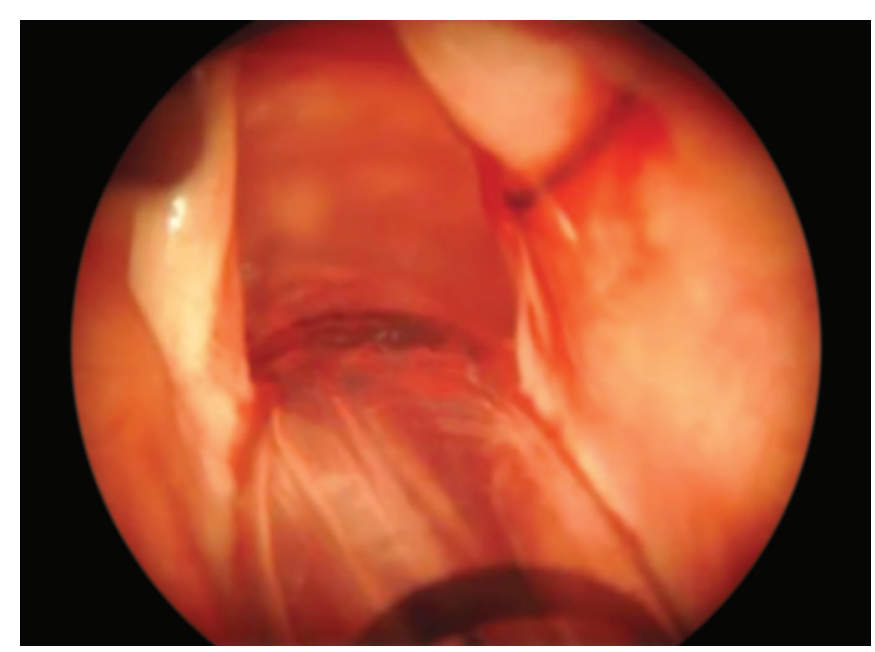

Fig. 4: Intraoperative view after application of suture and cord lateralization

the outside. The loop around the vocal fold is pulled until it gets sufficiently lateralized. The intraoperative final appearance of the lateralization of vocal cord appears as mentioned in Figure 4. The ends of the suture were tied externally by eight knots and then buried subcutaneously. The complications associated with this procedure were analyzed during surgery and immediately during the follow-up. Postoperatively the results of this surgery were analyzed. The lateralization of the vocal cord appears as mentioned in Figure 5 during the postoperative period allowing sufficient space for breathing with an acceptable social voice.

\section{OBSERVATIONS AND RESULTS}

In the present study, we have performed suture lateralization of vocal cords in 17 patients. Of these patients, $11(64.70 \%)$ were female and 5 (29.41\%) were male with a slight female preponderance. The mean age of performing the procedure was 39 years with the patients in the age group of 18 to 60 years. When the clinical etiology of the patients leading to bilateral vocal cord palsy was observed, $47.05 \%$ patients presented with postsurgical causes as documented in Table 1.

In the present study, it was observed that the average time for performing the procedure in postsurgical group of patients was 15.8 days, whereas it was 96.11 days in patients with systemic neurological diseases. Of these patients, tracheostomy was performed as an emergency procedure in 7 (41.17\%) patients. Rest of the patients were

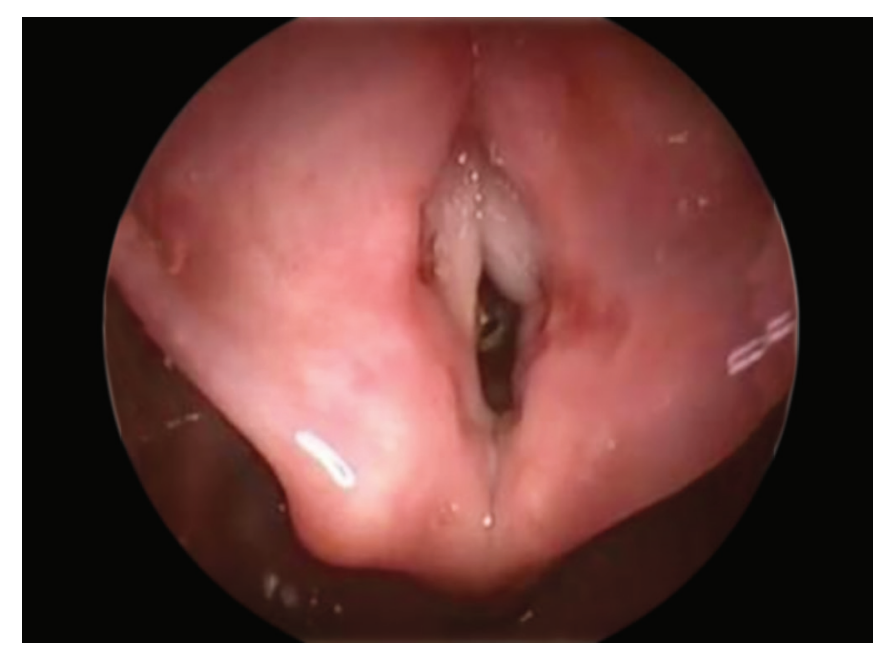

Fig. 5: Postoperative status of vocal cord with cord being lateralized

managed with endotracheal intubation. Tracheostomy closure was done in 6 out of 7 patients after 2 weeks following the procedure during the postoperative period.

Patients were followed up regularly on a monthly basis, and the median postoperative follow-up period for the patients was 6 months. During the study, it was observed that improvement in respiration was adequate in $16(94.11 \%)$ patients, and voice quality was socially acceptable in all the $16(94.11 \%)$ patients. The procedure failed to achieve a satisfactory laryngeal airway in only one patient, in whom decannulation of the tracheotomy was not achieved. When the complications of the procedure were observed, edema was observed in all the patients postoperatively which was symptomatically managed and subsided over a period of 48 hours. During the study, no major life-threatening complications like granulomas, keloid formations, aspiration, or cutting through the cord were recorded.

\section{DISCUSSION}

Voice production is one of the most fundamental functions after pathway of respiration. Laterofixation of the vocal fold is a simple and reliable surgical intervention for laryngeal obstruction and improvement of voice. ${ }^{5}$ The characteristic symptoms of bilateral abductor vocal cord paralysis include stridor with near-normal voice. However, the quality of voice may worsen during an acute stage of the disease. These symptoms may be attributed to the stationary and flaccid midline position

Table 1: Number of patients with sex distribution and etiology of vocal cord paralysis with tracheostomy performed

\begin{tabular}{llll}
\hline Etiology & Number of patients & Duration of illness & Tracheostomy performed \\
\hline Postsurgical & $8(5 \mathrm{~F}, 3 \mathrm{M})(47.05 \%)$ & $<3$ months & Not done \\
Systemic neurological diseases & $7(6 \mathrm{~F}, 1 \mathrm{M})(41.17 \%)$ & $>3$ months & Done \\
Idiopathic & $2(2 \mathrm{M})(11.76 \%)$ & $>3$ months & Done \\
\hline
\end{tabular}


of the vocal cords which places them in a phonating position, where they both obstruct the airway and produce a fairly clear voice. ${ }^{6}$ In the present study, Prolene is passed through the needles and the needles are then withdrawn. The thread thus forms a permanent loop around the vocal cord and creates space allowing for normal breathing with acceptable social voice.

Other techniques like resections or transections of anatomical structures within the larynx are also employed for restoration of voice and for further normalcy of the patient. These methods commonly involve arytenoidectomy, chordotomy, and cordectomy which reshape the glottis by means of shifts of existing anatomical structures. ${ }^{7}$ However, the disadvantage of these procedures is that the changes associated with these procedures are often permanent and irreversible. Other experimental procedures like artificial laryngeal pacing, ${ }^{8}$ injections of botulinum toxin ${ }^{9}$ into the cricothyroid muscle are being practiced; however, further research in this area is required.

It is often difficult to differentiate between paralysis and cartilage fixation from history or from observation and may indeed be indiscernible even by videostroboscopy. Testing the passive mobility of the arytenoids is important to differentiate between these two conditions, as the treatment options are different for these two conditions.

We suggest our method of laterofixation to be the preferred treatment in patients suffering from bilateral vocal cord paralysis where there is still a possibility of recovery of nerve function. Following suture lateralization there is usually minimal bleeding, scarring, and it is considered as safe alternative technique to tracheostomy over the other procedures. The advantage of performing surgery by our technique is that this procedure is reversible to a large extent and gives good results of the postoperative voice. Surgically, this procedure is minimally invasive with minimal alterations to the laryngeal mucosa. The most significant feature is that recovery of cord mobility and functionality can be expected following this procedure. Also, if needed, there is a possibility for performing extensive surgical procedure at a later time to the patient.

\section{CONCLUSION}

The surgery of laterofixation can provide immediate and long-lasting adequate airway. It can be considered as minimally invasive with minimal alterations to the laryngeal mucosa. It is also potentially reversible from the point of view of laryngeal functions, and thus subsequently preserves laryngeal functions. Thus, this procedure appears as a reliable treatment in the management of bilateral vocal cord palsy.

\section{REFERENCES}

1. Kort Y, Kessentini N, Khammassi N, Abdelhedi H. Twelfth nerve palsy in Sjogren's syndrome. Br J Med Pract 2016 Dec;9(4):a933.

2. Franco RA, Andrus JG. Common diagnoses and treatments in professional voice users. Otolaryngol Clin North Am 2007 Oct;40(5):1025-1061.

3. Grist W. Subglottic and tracheal stenosis in adults. In Encyclopedia of otolaryngology, head and neck surgery, editor Kountakis SE, Berlin: Springer; 2013. pp. 2594-2598.

4. Ejnell H,Mansson I, Bake B, Hallén O, Stenborg R, Lindstroum J. A simple operation for bilateral vocal cord paralysis. Laryngoscope 1984 Jul;94(7):954-958.

5. Jori J, Rovo L, Czigner J. Vocal cord laterofixation as early treatment for acute bilateral abductor paralysis after thyroid surgery. Eur Arch Otorhinolaryngol 1998 Aug;255(7):375-378.

6. Daya H, Hosni A, Bejar-Solar I, Evans JN, Bailey CM. Pediatric vocal fold paralysis: a long-term retrospective study. Arch Otolaryngol Head Neck Surg 2000 Jan;126(1):21-25.

7. Sapundzhiev N, Lichtenberger G, Eckel HE, Friedrich G, Zenev I, Toohill RJ, Werner JA. Surgery of adult bilateral vocal fold paralysis in adduction: history and trends. Eur Arch Otorhinolaryngol 2008 Dec;265(12):1501.

8. Broniatowski M, Kaneko S, Jacobs G, Nose Y, Tucker HM. Laryngeal pacemaker. II. Electronic pacing of reinnervated posterior cricoarytenoid muscles in the canine. Laryngoscope 1985 Oct;95(10):1194-1198.

9. Cohen SR, Thompson JW. Use of botulinum toxin to lateralize true vocal cords: a biochemical method to relieve bilateral abductor vocal cord paralysis. Ann Otol Rhinol Laryngol 1987 Sep-Oct;96(5):534-541. 